\title{
The self-inflicted burns-Typology and its prognostic relevance in a 14-year review of self-inflicted burns in a tertiary referral centre
}

\author{
Paula Moniz, Diogo Casal ${ }^{*}$, Carlos Mavioso, José Videira-Castro, Maria Angélica-Almeida \\ Burn Unit and Plastic and Reconstructive Surgery Department, São José Hospital, Rua José António Serrano, 1150-199 Lisbon, Portugal
}

\section{A R T I C L E I N F O}

Article history:

Accepted 22 July 2010

\section{Keywords:}

Self-inflicted burns

Suicide attempt

Psychiatric disorder

Self-injurious behavior

\begin{abstract}
A B S T R A C T
Self-inflicted burns (SIB) are responsible for $2-6 \%$ of admissions to Burn Units in Europe and North America, and for as many as $25 \%$ of admissions in developing nations. Recently, a promising new tool was proposed to stratify SIB patients in the following subgroups: "typical", "delirious", and "reactive". However, as far as the authors know, the clinical usefulness of this instrument has not yet been validated by others.

We retrospectively reviewed the clinical records of 56 patients admitted to our Burn Unit with the diagnosis of SIB injury in the past 14 years. The following parameters were evaluated: demographic features; psychiatric illness; substance abuse; mechanism of injury; burn depth, total body surface area (TBSA) involved, Abbreviated Burn Severity Index (ABSI); length of hospital stay, and mortality. All patients were followed up by a psychologist and a psychiatrist, and were classified according to the SIB-Typology Tool, into three classes: "typical", "delirious" and "reactive".

There was a slight predominance of the "typical" type (44.6\%), followed by the "delirious" type (30.4\%), and, finally the "reactive" type (25.0\%). Mortality was significantly higher in the "typical" subgroup. In conclusion, the SIB-Typology Tool appears to be a valuable instrument in the clinical management of SIB patients.
\end{abstract}

(C) 2010 Elsevier Ltd and ISBI. All rights reserved.

\section{Introduction}

Although accounts of culturally approved ritualistic selfimmolation go back as far as the 1st century BC in ancient Greece [1], suicide by self-burning is still one of the most perplexing human acts [2-4]. This method of suicide is reported most frequently in developing nations, being rather rare in developed countries [3]. In fact, in developing countries, like Iran, India, and Sri Lanka, it represents as many as $27 \%$ of all suicide attempts [2,5,6].

However, even in developed countries, it corresponds to approximately $1 \%$ of all suicides $[5,7,8]$. Consequently, selfimmolation is responsible for $2-6 \%$ of admissions to Burn
Units in Europe and North America, and for as many as $25 \%$ of admissions in developing nations [9-16]. To make matters even worse, it has been clearly shown that intentional burn patients have a larger mean total body surface area (TBSA) burned, a longer hospital stay, and a higher mortality compared to other burned patients [17]. In some series, mortality in these patients reaches an astounding $80 \%[3,18]$.

In spite of this, knowledge of self-inflicted burns (SIB) is still generally considered to be sparse [2]. In Western countries, SIB injuries have been associated with previous psychiatric disorders or predisposing factors such as: substance abuse, relationship problems, unemployment and significant emotional trauma $[10,14]$. In opposition, in low-income countries,

\footnotetext{
* Corresponding author at: Rua Luis Pastor de Macedo, N32, 5D, 1570-159 Lisbon, Portugal. Tel.: +351 916117315.

E-mail address: diogo_bogalhao@yahoo.co.uk (D. Casal). 
SIB is frequently linked to suicide attempts $[2,18,19]$. The high rates of suicide attempts by SIB in these regions have been attributed to several factors, namely: the 'dowry' system [2,19-21], the influence of Ancient Greeks [22], religious influences [23,24], sanguine marriages [25], the traditional style of Kurdish women's clothing [26], and even the possible contamination with natural sour gas containing hydrogen sulfide [27]. Not surprisingly, several authors have proposed that Public Health measures to curb the incidence of SIB must be based on a deep knowledge of the risk factors associated with SIB in specific populations $[2,5,6,19,20,28]$. By using this strategy, Ahmadi and Ytterstad, for example, were able to reduce the mean self-immolation attempts rate by $57 \%$ in the Iranian city of Gilangharb [6]. In fact, it is now widely accepted that, if we are to reduce mortality associated with this type of self-aggression, a sounder grasp of the cultural, psychological and psychiatrical motivations of SIB is urgently needed [2,4].

Recently, a promising new tool was proposed by Titscher et al. to stratify SIB patients in three different subgroups. This instrument was named self-inflicted burns (SIB)-Typology Tool, and its aim was to aid in the management of SIB patients [9]. In fact, Titscher et al. noted that these three subgroups seemed to be associated with distinct patterns of mechanism of burns, evolution during hospital stay, medical outcome and social reintegration [9]. Therefore, the SIB-Typology Tool holds great promise as a means to better characterize and understand SIB patients. Additionally, this instrument may serve as an adjunct to clinical decision making, namely on possible discharge options for different subsets of SIB patients [9].

However, the SIB-Typology Tool was developed based on the experience with the treatment of no more than 36 SIB patients, and its clinical usefulness was evaluated prospectively in only 6 patients. In addition, all the data was obtained from the same Burn Unit, which may hamper generalization to other populations. Moreover, the clinical utility of this instrument in this context has not yet been replicated by others.

Therefore, the aim of this study was to assess the clinical usefulness of the SIB-Typology Tool in the management of SIB patients in a larger series of patients from a different population.

\section{Methods}

\subsection{Procedure}

We retrospectively reviewed the clinical records of all patients admitted to our Burn Unit in the period between May 25th 1995 and April 30th 2009 who had sustained a SIB injury. This Burn Unit is currently the largest in the Portugal, receiving patients from all over the country, but particularly from the south regions. On average, around 100 patients are admitted to this Burn Unit each year.

All the information was collected independently from the patients' clinical charts by the two first two authors (P.M. and D.C.). The data collected by each of these authors was then compared. When discrepancies were found, the clinical charts were reviewed by all the authors.

\subsection{Inclusion and exclusion criteria}

SIB patients were identified based on unequivocal information provided by either the paramedics and/or doctors that brought the patient to the hospital and/or by the patient him/herself. If in doubt about the existence of SIB injury, patients were excluded from the study.

\subsection{Measurements}

The following parameters were evaluated:

- demographic features;

- (pre-) existing psychiatric illness;

- substance abuse;

- mechanism of injury;

- burn depth, total body surface area (TBSA) burned and Abbreviated Burn Severity Index (ABSI) [29];

- length of hospital stay;

- mortality.

All patients were evaluated and followed up by a psychologist and a psychiatrist. Based on their detailed notes in the medical records, the first two authors (P.M. and D.C.) independently classified each patient according to the selfinflicted burns (SIB)-Typology, into three classes: "typical", "delirious" and "reactive" [9].

Succinctly, the SIB-Typology classes can be described in the following manner:

- the "typical" SIB patient has usually a known psychiatric history, and has frequently planned or attempted to commit suicide in the past;

- the "delirious" SIB patient harms him/herself under the influence of substance abuse and/or psychotic episode, and not as a deliberate and insightful intent of killing him/ herself;

- the "reactive" SIB patient tries to commit suicide as the result of a significantly negative life event, which the patient feels unable to cope with; many of the patients in this group have no history of psychiatric diseases, and act under the influence of alcohol and/or drugs [9].

\subsection{Analyses}

Statistical analysis was performed using the Statistical Analysis Software PASW Statistics $18\left(\mathrm{IBM}^{\mathbb{R}}\right)$. Chi-Square test was used to compare proportions. A t-Student and ANOVA were used to compare means, as indicated. A $p$ value under 0.05 was considered statistically significant. All mean values are expressed as the mean value \pm standard deviation.

\section{Results}

In the 14-year review we conducted, we identified 56 patients that had sustained burns as a result of self-aggression. This group represented $4.4 \%$ of the global number of patients admitted to our Burn Unit in that period (1283 patients). The 
SIB group was composed of 36 men (64.3\%) and 20 women (35.7\%).

The average age in the SBI was $50.4 \pm 19.3$ years, ranging from 22 to 89 years. Mean age was higher in the male group, but this difference was not statistically significant $(53.3 \pm 20.0$ years vs. $45.0 \pm 17.4$ years).

The written evaluation in the clinical charts, performed by the psychologist and the psychiatrist that evaluated the SIB patients both in the Burn Unit and after discharge, concurred in all cases. A prior psychiatric history was ascertained in $67.9 \%$ of SIB cases (38 cases). This value was significantly higher than that reported for the general population of patients admitted to the Burn Unit in the same time period, which was only $9.5 \%(p<0.01)$. Among the several prior psychiatric diagnoses in the SIB patients, the most frequent were: depression (33.3\%), schizophrenia (22.2\%), and mental retardation (22.2\%). Over a quarter of patients (28.6\%) reported substance abuse, which was significantly more than the $11.8 \%$ of patients with identified drug abuse in the general Burn Unit population in the same period ( $p<0.05$ ). In $17.9 \%$ of cases of SIB there was a history of drug abuse and in $10.7 \%$ of cases of alcohol addiction.

Most patients attempted suicide by pouring an inflammable fluid over themselves, followed by its ignition, or by setting their clothes or surroundings on fire directly (92.9\%). A minority tried to commit suicide through contact with electricity (3.6\%) or by pouring down acid over their bodies (1.8\%). In one patient the exact cause of the burn could not be determined, as the patient died a few hours after admission.

All patients had deep partial or full thickness burns. The mean TBSA was $32.2 \% \pm 25.1$. However, mean TBSA was significantly higher for men than for women $(37.2 \% \pm 27.7$ vs. $23.2 \% \pm 16.6 ; p<0.05)$. The mean ABSI was $5.2 \pm 2.6$, with an average of $5.4 \pm 3.0$ for males and $4.8 \pm 1.8$ for women. However, this difference was not statistically significant.

The classification of SIB patients according to SIB-Typology [9], performed independently by the first two authors based on the psychologist and the psychiatrist's notes in the clinical charts, matched in all cases. Distribution of patients according to SIB-Typology is depicted in Fig. 1. There was a slight predominance of the "typical" type (44.6\%), followed by the "delirious" type (30.4\%), and, finally the "reactive" type (25.0\%). Regarding gender, males were over-represented in the "delirious" and "reactive" groups, whereas in the "typical" category there was a slight female predominance (Fig. 2).

The mean length of hospitalization was $24.8 \pm 19.4$ days, ranging from 1 to 90 days. Mortality in the SIB group was $42.8 \%$, which was significantly higher than that observed in the general population of patients admitted to the Burn Unit in the same period of time (13.9\%; $p<0.01)$. No significant difference was found between the length of hospital stay in the different SIB subgroups. However, mortality was significantly higher in the "typical" category of SIB patients (60.0\%) compared with the "reactive" (42.8\%) and "delirious" (17.6\%) subgroups $(p<0.05$; Fig. 3).

For each SIB category, males had more extensive burns (Fig. 4). Notwithstanding, this did not result in a significantly higher mortality in males. In addition, the differences between TBAS and ABSI in the different SIB categories were also not statistically significant.
The Self-Inflicted-Burns-Typology (SIB-T)

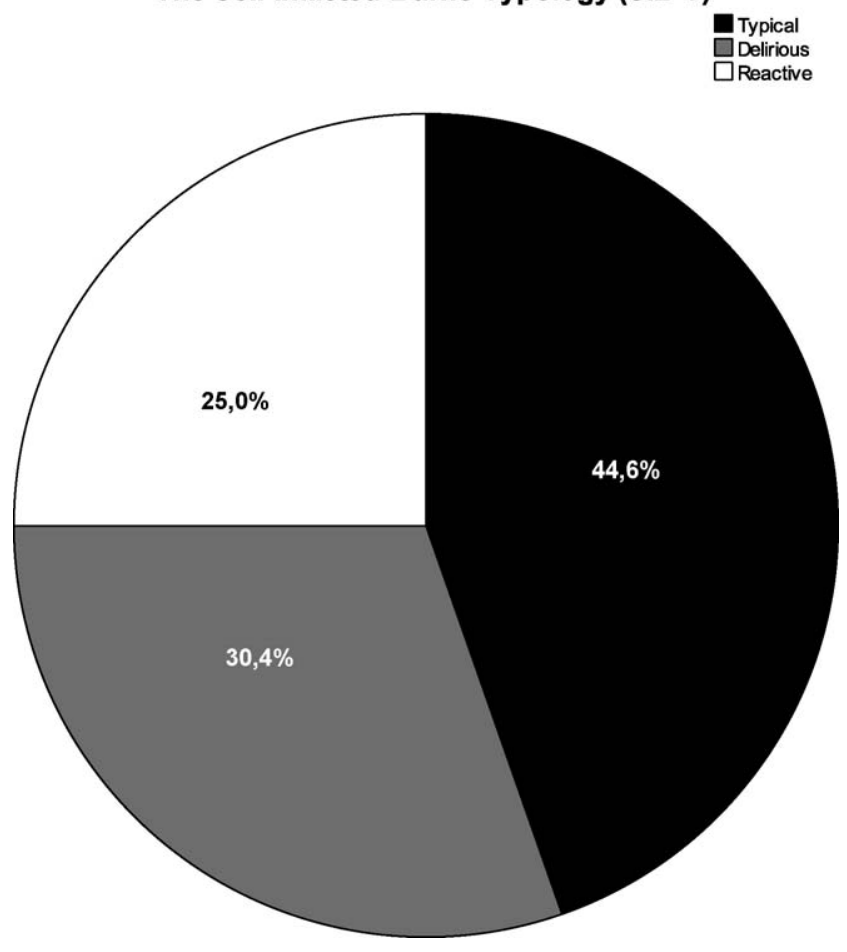

Fig. 1 - Distribution of patients with self induced burns according to the SIB-Typology proposed by Titscher et al. [9].

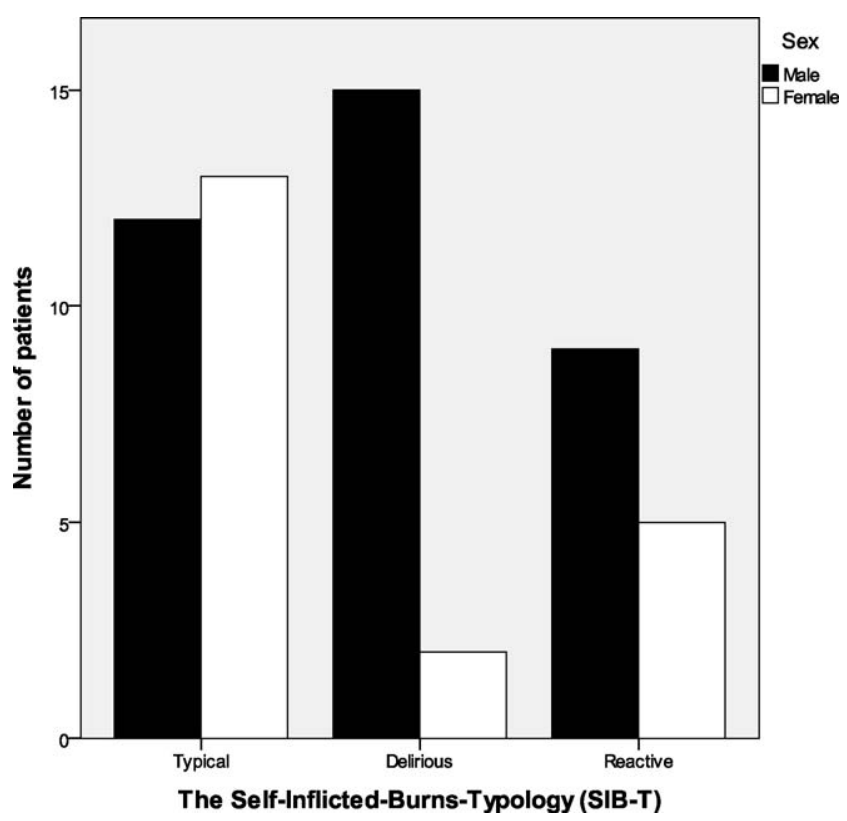

Fig. 2 - Distribution of patients with self induced burns according to gender and SIB-Typology.

\section{Discussion}

Reviewing the literature on SIB, we find that our results do not generally differ from what has generally been reported $[9,10,12,30,31]$. Interestingly, as most authors, we found a higher TBSA involved, a higher mortality, and higher rates of 


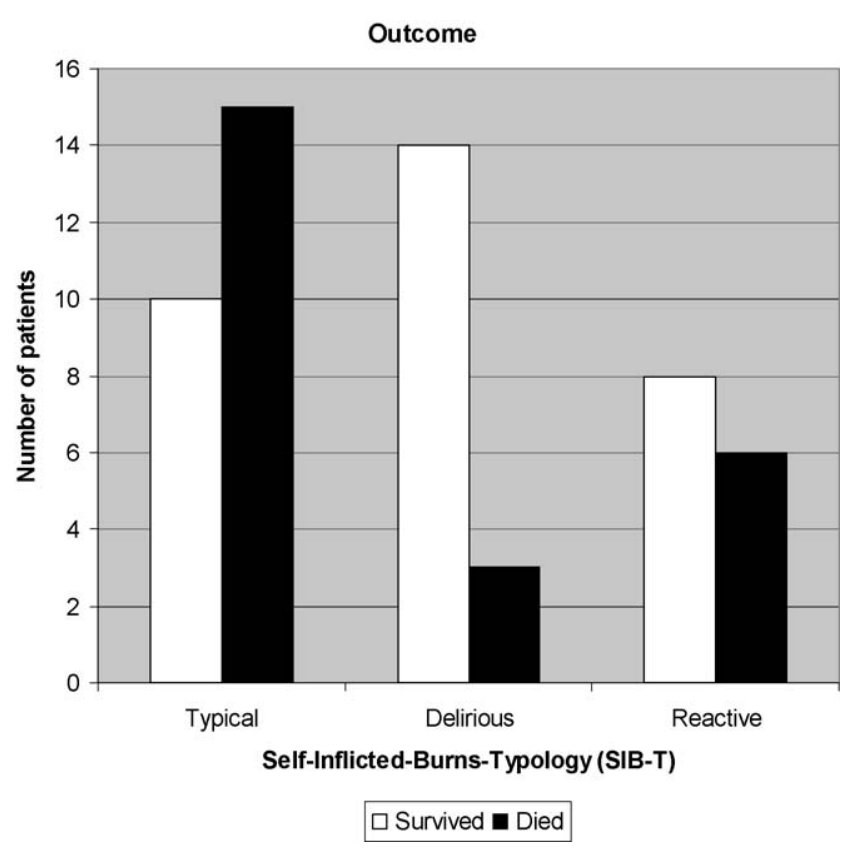

Fig. 3 - Outcome in the different SIB groups. Mortality was significantly higher $(p<0.05)$ in the "typical" category of SIB patients compared with the "reactive" and "delirious" subgroups.

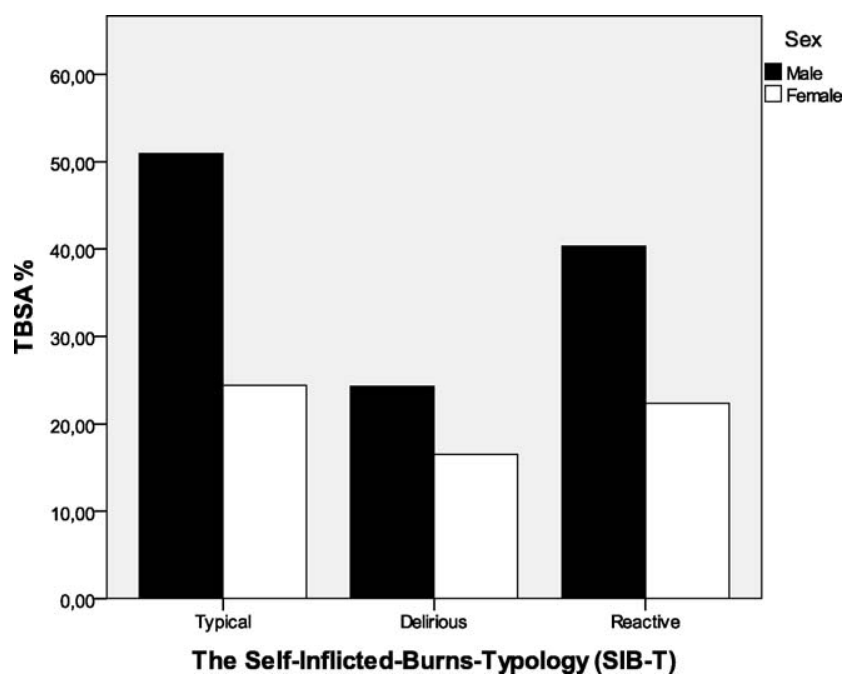

Fig. 4 - Total body surface area (TBSA) involved in the different SIB groups. For each SIB category, males had more extensive burns $(p<0.05)$.

previous psychiatric disease and substance abuse in the SIB group compared with the general population in the Burn Unit $[10,17]$. This lends consistency to the notion that SIB patients' demographic and clinical features are remarkably consistent in Western European and North American countries [9,12,31].

As far as the authors know, this was the first time, since its conception, that the SIB-Typology Tool was used systematically for evaluating SIB patients [9]. In our experience this was an easy-to-use instrument that did in fact help in stratifying patients with SIB in relatively homogenous subgroups. The fact that the classification of SIB patients according to SIB-
Typology, performed independently by the first two authors, matched in all cases, seems to indicate that this classification is reproducible.

Interestingly, the differentiation of SIB patients into "typical", "reactive", and "delirious", may well lead to a revision of what is "typical" and "non-typical" in this population of patients. In fact, in our series, most patients $(55.4 \%)$ were in the non-typical subgroups, that is to say the "reactive", and "delirious" subgroups (25.0\% and $30.4 \%$, respectively). A similar observation was made in the original work of Titscher et al. that in 6 SIB patients evaluated prospectively, fitted 3 into the "reactive" and 1 into the "delirious" categories.

Moreover, in our work, the SIB-Typology proved to be a valuable tool to evaluate clinical outcome. In fact, the inclusion alone of SIB patients in the "typical" subgroup was associated with a significantly higher mortality rate compared to the "reactive" and "delirious" subgroups. This association was independent of the TBSA involved and the ABSI, which were not significantly different between the three subgroups. However, no differences were found between the different subgroups regarding length of stay in the Burn Unit. Notwithstanding, this last observation may simply be due to the relatively small number of patients assessed in the present study.

Interestingly, the higher mortality rate observed during hospitalization in the "typical" subgroup is in agreement with what is known about the impact of psychiatric illness in burn patients. In fact, the "typical" SIB patient is characterized by a known psychiatric history [9]. In addition, it is clearly established that previous psychiatric illnesses are associated not only with poor motivation and non-compliance with therapeutic strategies, but also with delayed wound healing [9,31-33]. Therefore, it is hardly surprising that the "typical" subgroup was associated with a grimmer prognosis during Burn Unit stay. In contrast, both "reactive" and "delirious" SIB patients are by definition exempt of protracted psychiatric diseases. As a result, after having been confronted with their own possible death, they are frequently eager to resume their normal life, and are, consequently, keen on collaborating with their treatment [9]. This may be the reason why their outcome is better.

Hence, the SIB-Typology seems to have two significant merits for the management of SIB patients in the Burn Unit: on the one hand, it seems to have prognostic significance; on the other hand, it may identify a subgroup of SIB patients (the "typical" subgroup) in which a more intensive psychological and psychiatric treatment may lead to a better medical outcome.

The SIB-Typology may also be of great interest in aiding decisions regarding discharge or need of subsequent psychiatric help [9]. As it was noted by Titscher et al., "typical" SIB patients not only lack motivation and willingness to comply with therapeutics, but also have a poorer family and social support, as well as a lower economical status due to their lingering psychiatric illness [9,34]. In opposition, "delirious" and "reactive" patients have usually a better social, economical and family support [9]. Therefore, it seems sensible to recommend that "typical" SIB patients are usually sent to a psychiatric clinic after discharge from the Burn Unit, whereas 
most "reactive" and "delirious" SIB patients could be discharged home, and followed up in a Psychiatry outpatient clinic [9].

From a Public Health point of view, the SIB-Typology Tool, by allowing the identification of coherent subgroups among the diverse population of SIB patients, may be of paramount importance in identifying risk and protective factors for each subgroup. This, in turn, may be instrumental in developing prevention strategies $[6,18]$. For example, the recognition that in our series only $44.6 \%$ of SIB patients fitted in the "typical" subgroup may suggest that prevention efforts should be made to address an until now underestimated fraction of SIB patients: the "delirious" and "reactive" patients. In this sense, it might make sense to expose substance abusers or people undergoing distressful life events, for instance, to videos showing SIB victim stories, in order to prevent SIB injuries in these groups of patients [6].

However, we must note that care should be exercised while extrapolating the results of the present study. In fact the relatively small number of SIB patients assessed, the retrospective nature of the study, and the fact that the study was conducted in a single Burn Unit serving a specific population may hamper the generalizability of the results to other populations and contexts. Hence, prospective studies, involving a larger number of patients, from different countries, are warranted to definitively validate the usefulness of the SIBTypology in the clinical setting.

In conclusion, the SIB-Typology Tool appears to be a valuable instrument in clinical decision making, and for delineating pre-emptive strategies in the realm of SIB patients. However, more studies are needed, to definitively determine its usefulness in this context.

\section{Conflict of interest}

The authors have no conflict of interest to declare in relation to the content of this article.

\section{R E F E R E N C E S}

[1] Crosby K, Rhee JO, Holland J. Suicide by fire: a contemporary method of political protest. Int J Soc Psychiatry 1977;23:60-9.

[2] Ahmadi A, Mohammadi R, Schwebel DC, Hassanzadeh M, Yari M. Classic philosophy lessons and preventing selfinflicted burns: a call for action. Burns 2009;35:154-5.

[3] Ahmadi A, Mohammadi R, Schwebel DC, Yeganeh N, Hassanzadeh M, Bazargan-Hejazi S. Psychiatric disorders (Axis I and Axis II) and self-immolation: a case-control study from Iran. J Forensic Sci 2010;55:447-50.

[4] Thombs BD, Bresnick MG, Magyar-Russell G. Who attempts suicide by burning? An analysis of age patterns of mortality by self-inflicted burning in the United States. Gen Hosp Psychiatry 2007;29:244-50.

[5] Ahmadi A, Mohammadi R, Schwebel DC, Yeganeh N, Soroush A, Bazargan-Hejazi S. Familial risk factors for selfimmolation: a case-control study. J Womens Health (Larchmt) 2009;18:1025-31.

[6] Ahmadi A, Ytterstad B. Prevention of self-immolation by community-based intervention. Burns 2007;33:1032-40.
[7] Cave Bondi G, Cipolloni L, Parroni E, Cecchi R. A review of suicides by burning in Rome between 1947-1997 examined by the Pathology Department of the Institute of Forensic Medicine, University of Rome 'La Sapienza'. Burns 2001;27:227-31.

[8] Rothschild MA, Raatschen HJ, Schneider V. Suicide by selfimmolation in Berlin from 1990 to 2000. Forensic Sci Int 2001;124:163-6.

[9] Titscher A, Lumenta DB, Belke V, Kamolz LP, Frey M. A new diagnostic tool for the classification of patients with selfinflicted burns (SIB): the SIB-Typology and its implications for clinical practice. Burns 2009;35:733-7.

[10] Malic CC, Karoo RO, Austin O, Phipps A. Burns inflicted by self or by others-an 11 year snapshot. Burns 2007;33:92-7.

[11] Rashid A, Gowar JP. Self-inflicted burns: a sporadic phenomenon. Burns 2004;30:833-5.

[12] Hadjiiski O, Todorov P. Suicide by self-inflicted burns. Burns 1996;22:381-3.

[13] Pham TN, King JR, Palmieri TL, Greenhalgh DG. Predisposing factors for self-inflicted burns. J Burn Care Rehabil 2003;24:223-7.

[14] Greenbaum AR, Donne J, Wilson D, Dunn KW. Intentional burn injury: an evidence-based, clinical and forensic review. Burns 2004;30:628-42.

[15] Laloe V. Patterns of deliberate self-burning in various parts of the world. A review. Burns 2004;30:207-15.

[16] Laloe V. Epidemiology and mortality of burns in a general hospital of Eastern Sri Lanka. Burns 2002;28:778-81.

[17] Modjarrad K, McGwin Jr G, Cross JM, Rue 3rd LW. The descriptive epidemiology of intentional burns in the United States: an analysis of the National Burn Repository. Burns 2007;33:828-32.

[18] Ahmadi A, Mohammadi R, Stavrinos D, Almasi A, Schwebel DC. Self-immolation in Iran. J Burn Care Res 2008;29:451-60.

[19] Ahmadi A. Suicide by self-immolation: comprehensive overview, experiences and suggestions. J Burn Care Res 2007;28:30-41.

[20] Ahmadi A, Mohammadi R, Schwebel DC, Khazaie H, Yeganeh N, Almasi A. Demographic risk factors of selfimmolation: a case-control study. Burns 2009;35:580-6.

[21] Menezes RG, Kanchan T, Pillay VV, Lobo SW, Subba SH. Self-inflicted burns. Burns 2008;34:427.

[22] Norbury WB, Jeschke MG, Herndon DN. Suicidal intention and self-immolation: what is the outcome? Crit Care Med 2008;36:345-6.

[23] Grossoehme DH, Springer LS. Images of God used by selfinjurious burn patients. Burns 1999;25:443-8.

[24] Grossoehme DH. Prayer reveals belief: images of God from hospital prayers. J Pastoral Care 1996;50:33-9.

[25] Saadat M, Zendeh-Boodi Z. Correlation between incidences of self-inflicted burns and means of inbreeding coefficients, an ecologic study. Ann Epidemiol 2006;16:708-11.

[26] Groohi B, Alaghehbandan R, Lari AR. Analysis of 1089 burn patients in province of Kurdistan, Iran. Burns 2002;28:569-74.

[27] Saadat M, Bahaoddini A, Mohabatkar H, Noemani K. High incidence of suicide by burning in Masjid-i-Sulaiman (southwest of Iran), a polluted area with natural sour gas leakage. Burns 2004;30:829-32.

[28] P.H.S. U.S. Department of Health and Human Services, editor. National strategy for suicide prevention: goals and objectives for action. Rockville, MD: U.S. Surgeon General; 2001.

[29] Tobiasen J, Hiebert JM, Edlich RF. The Abbreviated Burn Severity Index. Ann Emerg Med 1982;11:260-2.

[30] Antonowicz JL, Taylor LH, Showalter PE, Farrell KJ, Berg S. Profiles and treatment of attempted suicide by selfimmolation. Gen Hosp Psychiatry 1997;19:51-5.

[31] Ali SN, Soueid A, Rao K, Moiemen N. Self-inflicted burns, outcome and cost. Burns 2006;32:463-6. 
[32] Kamolz LP, Andel H, Schmidtke A, Valentini D, Meissl G, Frey M. Treatment of patients with severe burn injuries: the impact of schizophrenia. Burns 2003;29:49-53.

[33] Tarrier N, Gregg L, Edwards J, Dunn K. The influence of preexisting psychiatric illness on recovery in burn injury patients: the impact of psychosis and depression. Burns 2005;31:45-9.

[34] Nettelbladt P, Svensson C, Serin U, Ojehagen A. The social network of patients with schizoaffective disorder as compared to healthy individuals. Soc Sci Med 1995;41:901-7. 